\title{
The Protective Effects of Protein-Enriched Fraction from Housefly (Musca domestica) against Aged-Related Brain Aging
}

\author{
Yanan TANG ${ }^{1,2}$, Panpan $\mathrm{FENG}^{3}$, Shuiqing GuI ${ }^{4}$, Xiaobao $\mathrm{JIN}^{1,2}$, \\ Jiayong ZHU ${ }^{1,2}$ and Xuemei $\mathrm{LU}^{1,2, *}$ \\ ${ }^{1}$ School of Life Science and Biopharmaceutics, Guangdong Pharmaceutical University, \\ Guangzhou 510006, P.R. China \\ ${ }^{2}$ Guangdong Provincial Key Laboratory of Pharmaceutical Bioactive Substances, \\ Guangdong Pharmaceutical University, Guangzhou 510006, P.R. China \\ ${ }^{3}$ Guangdong Lingnan Institute of Technology, Guangzhou 510663, P.R. China \\ ${ }^{4}$ Intensive Care Unit, Shenzhen Second People's Hospital, The First Affiliated Hospital of \\ Shenzhen University, Shenzhen 518031, P.R. China
}

(Received April 14, 2019)

\begin{abstract}
Summary The Musca domestica larvae are well known for its multifunctions and great nutritional value. The present study aimed at investigating the beneficial effect of Musca domestica larvae extract (Mde) against memory impairment, structural damage and oxidative stress in aged rats. Twenty-month-old rats were gavaged with Mde for 2 mo. Morris Water Maze test indicated Mde prevented aging-induced spatial learning and memory dysfunction in the aged rats. Mde supply was also found to attenuate age-associated changes of brain histology that observed by light microscopy and transmission electron microscopy. Moreover, the increase of antioxidant capacity, glutathione peroxidase (GPx) activity, superoxide dismutase (SOD) activity, as well as the decreased methane dicarboxylic aldehyde (MDA) levels, were consistent with these results. Hence, we propose that oral administration of Mde could improve memory impairment via antioxidant action, and Mde has the potential to act as an excellent food supplement or medicine for the attenuation of brain aging.

Key Words Musca domestica larvae extract, antioxidants, aged rats, oxidative damage, memory impairment
\end{abstract}

The aged tendency of population is a major public health problem and one of the most complex issues in neuroscience today, which results in increasing public health concerns and health expenditures $(1,2)$. It is a widely accepted consensus that aging is typically accompanied by gradual and progressive cognitive decline and pathophysiological changes in the brain, which may cause age-related neurodegenerative diseases such as Alzheimer's disease, Parkinson's disease and so on $(3,4)$. The free radical (FR) theory was put forward to demonstrate the aged-associated cognitive decline and neuronal loss may be caused by the cumulative of oxidative damage in cells and tissues (5). Numerous studies also proved that the antioxidant supplements is an important step in the prevention of aging, because the antioxidants can scavenge free radicals or oxidation products that bring about oxidative damage $(6,7)$.

Insects and insert-derived products are extensive and unexploited sources of potentially useful compounds for modern medicine (3). Musca domestica larvae have been used as a therapeutic drug in China since Ming dynasty. Previous study has revealed that the extract of Musca domestica larvae has the ability to elevate antioxidant

\footnotetext{
*To whom correspondence should be addressed.

E-mail: luxuemei605@163.com
}

enzyme activities in vitro (8). Hence, it was expected that Musca domestica larvae extract may improve brain health against oxidative damage. Nevertheless, we rarely found its anti-aging aspect reports on age-related cognitive impairment in natural aging rats.

In the present study, the natural aging rat (20-mo-old) was used as a model to study the effect of Musca domestica larvae extract (Mde) on aged-related memory impairment. Structural alterations of brain were analyzed by optical microscope and transmission electron microscopy (TEM). The activities of relative antioxidant factors were measured by biochemistry and molecular biology methods.

\section{MATERIALS AND METHODS}

Preparation of protein-enriched extract from Musca domestica larvae. Musca domestica larvae were obtained from the Guangdong Provincial Center for Disease Control and Prevention (CDC), China. The protein-enriched extract of Musca domestica larvae was prepared as described before (9). Briefly, the third-instar larvae were collected, washed with distilled water, and homogenized with a homogenizer ( $5 \mathrm{~s}$ at $3,500 \mathrm{rpm})$. Next, the homogenate was centrifuged at $1,300 \times \mathrm{g} / \mathrm{min}$ for $10 \mathrm{~min}$ to remove insoluble material. The obtained supernatant was concentrated and lyophilized. Then the lyophilized supernatant, an extract of Musca domes- 
tica larvae (Mde), was stored at $-80^{\circ} \mathrm{C}(10)$. The nutritional value of Mde has been determined in our previous studies $(9,11)$.

Animals and experimental design. All animal experiments were carried out according to the guidelines of the 3Rs (Replacement, Reduction and Refinement) after approval by the Ethical Committee for Research on Laboratory Animals of Guangdong Pharmaceutical University [China approval number SCXK (Yue) 20130002]. Adult female Sprague-Dawley (SD) rats (3 mo, 220 to $250 \mathrm{~g}$ ) and natural aging female SD rats (20 mo, 350 to $450 \mathrm{~g}$ ) were procured from Medical Laboratory Animal Center (Guangzhou, Guangdong, China). All rats were kept in specified pathogen free (SPF) conditions and maintained on a light/dark cycle (12 h/12 h) at $25 \pm 2^{\circ} \mathrm{C}$ with $55 \pm 10 \%$ relative humidity.

The rats were fed ad libitum with normal diet for $1 \mathrm{wk}$. Rats were fed with food provided by the Animal Center of Guangdong Pharmaceutical University (protein $22 \%$, fat $4.2 \%$, calcium $1.2 \%$, phosphorus $0.9 \%$, lysine $1.36 \%$, protein + cystine $0.82 \%$ ). After acclimatization, eight 3-mo-old SD rats were served as young control group. Forty 20-mo-old rats were randomly assigned to five groups: aged control group (normal saline, $n=8)$, vitamin $\mathrm{E}$ (VitE) group $(n=8)$, the lowdose Mde group (50 mg/kg, $n=8$ ), the middle-dose Mde group (100 mg/kg, $n=8$ ) and the high-dose Mde group $(200 \mathrm{mg} / \mathrm{kg}, n=8)$. All rats were given intragastric administration once a day at the same time, at the dose of $10 \mathrm{~mL} / \mathrm{kg}$ for $2 \mathrm{mo}$.

All rats were tested in Morris Water Maze after 2 mo. After the behavior test, all animals were deeply anesthetized with $2 \%$ sodium pentobarbital. For ultrastructural study, some rats' thoraces were opened, isotonic saline was quickly perfused into the aorta via left ventricle, and then ice cold $2.5 \%$ glutaraldehyde that had been diluted by $0.1 \mathrm{M}$ phosphate buffer were slowly perfused in the same way. Following perfusion, their brains were taken out immediately. In addition, the others' brains were rapidly removed, washed with ice-cold normal saline and stored at $-80^{\circ} \mathrm{C}$ till further analysis.

Behavioral study-Morris Water Maze (MWM). Behavior study was performed by Morris Water Maze test to observe the spatial learning and memory of rats (12). The test apparatus consisted of hidden escape platform (a $10 \mathrm{~cm}$ diameter round iron) and round pool (1.20 $\mathrm{m}$ diameter, $60 \mathrm{~cm}$ height), filled with water in the depth of $40 \mathrm{~cm}$ with temperature of $25 \pm 1^{\circ} \mathrm{C}$. Four equidistant points were designated as start positions and divided the pool into four quadrants. The hidden escape platform was placed under the water surface $2 \mathrm{~cm}$ in the center of southwest quadrant. Ink was poured into the water to make water turbid to prevent extra-maze cues (e.g. laboratory technician, table, window, cabinets, furniture and light). The test was carried out in a dark and sound proof room.

At the end of 60th days, rats were put into the pool 1 min before starting the experiment to allow habituation to the new environs. Behavior analysis was performed twice daily, lasting for $3 \mathrm{~d}$. The experimenter did not know the group of rats in the course of the experiment, so as to avoid the influence of subjective consciousness guidance on the experimental results. At the beginning of each test, rats were placed in the pool, facing each wall of the four quadrant edges in a pseudo-random manner. The rats were allowed to find the hidden escape platform in $60 \mathrm{~s}$, or they were gently guided to the platform by the experimenter. Then the rats were given a $10 \mathrm{~s}$ rest on the platform in the test interval time. The behavioral data were collected through a video camera fixed on ceiling and analyzed by the MNT-100 Video Tracking System (Chengdu Taimeng Tech. Co, Ltd, Chengdu, China).

Hematoxylin-eosin staining (HE). For histological analysis, brains were fixed with $4 \%$ paraformaldehyde for at least $24 \mathrm{~h}$, dehydrated in a series graded alcohols and embedded in paraffin. After which serial, $4 \mu \mathrm{m}$ slices were cut and transferred onto pre-treated glass slides. Sections were deparaffinized and stained with hematoxylin and eosin for examining the morphological changes in the brain. Sections were selected from all animals at the same level of the coronal section, one section was collected every 5 consecutive sections until a total of 5 sections had been acquired for each animal. Five visual fields $(\times 400)$ were randomly selected to count the numbers of preserved nerve cells per $1 \mathrm{~mm}^{2}$ in the cortical regions in a single-blinded manner by the Image J software (National Institutes of Health, Bethesda, MD, USA) (13).

Transmission electron microscopy. For brain ultrastructure observing, the brain were diced into blocks less than $1 \mathrm{~mm}^{3}$ and fixed in $2.5 \%$ glutaraldehyde. After further postfixation in fresh fixative $\left(1 \% \mathrm{OsO}_{4}\right)$ for $2 \mathrm{~h}$ with the temperature of $4^{\circ} \mathrm{C}$, the blocks were dehydrated in ascending series of alcohols and then embedded in epoxy resin for preparation of slices. The $0.5 \mathrm{~mm}$ thick slices were stained with $1 \%$ Toluidene blue and observed in an optical microscopy for further repairing the embedded block. Ultrafine sections were obtained by the use of an ultramicrotome with $1 \mathrm{~mm} / \mathrm{s}$ speed and $100 \mathrm{~nm}$ thickness. Finally, the ultrathin section were placed on a mesh with supporting film before staining Uranyl Acetate-Lead Citrate stain in a petri dish, to absorb the carbon dioxide at room temperature for $3 \mathrm{~min}$, the grid was removed and the slices were observed in a JEM1400 (Jeol, Tokio, Japan) transmission electron microscopy $(14,15)$. Micrographs were taken with a digital camera Bioscan 792 (Gatan Inc, Pleasanton, USA).

Measurement of biochemical indexes. The brains were homogenized by an organization dispersion machine (IKA, Germany). Next the homogenates were centrifuged at 3,000 rpm/min for $15 \mathrm{~min}$. Supernatant was collected for the measurement of oxidative stress indicators such as superoxide dismutase (SOD), glutathione peroxidase $(\mathrm{GPx})$ and methane dicarboxylic aldehyde (MDA). The level of SOD, GPx and MDA of rats in the brain homogenate was detected by the using the assay kits in accordance with specification requirements (Nanjing Jiancheng Bioengineering Institute, Nanjing, 

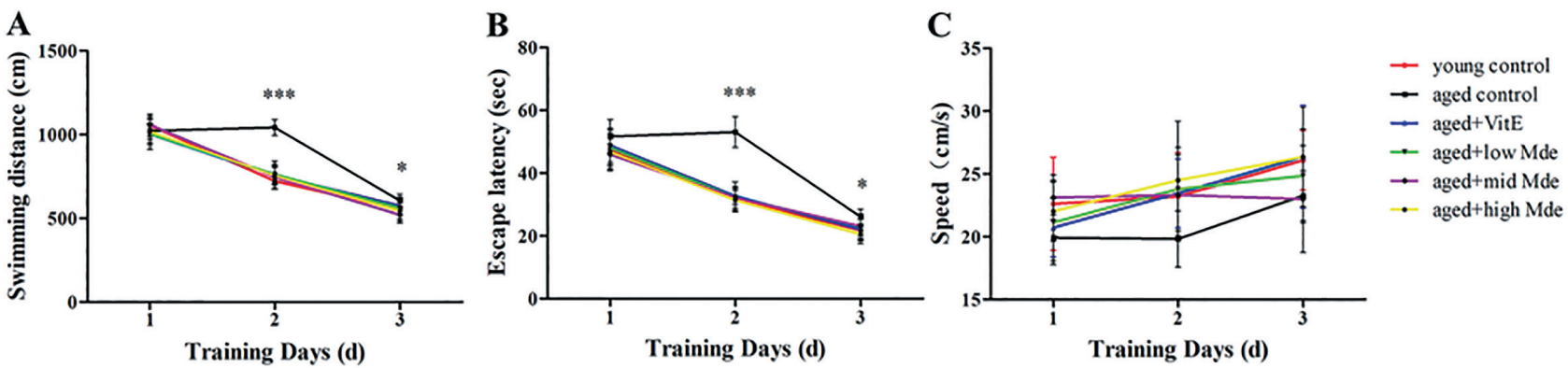

Fig. 1. Mde improved the learning and memory function in natural aging rats. The naturally-induced aging rats with and without the treatment of Mde was evaluated by Morris Water Maze. (A) The swimming distance of all groups to locate the hidden platform over $3 \mathrm{~d}$. (B) The escape latency of all groups to locate the hidden platform over $3 \mathrm{~d}$. (C) The swimming speed of all groups to locate the hidden platform over $3 \mathrm{~d}$. Data are represented as mean \pm SD. Compared with the aged control group, ${ }^{*} p<0.05,{ }^{* * *} p<0.001$.

China).

PCR and quantitative real-time PCR (QPCR). Total RNA was extracted from brain using an RNAiso plus (TaKaRa, Dalian, China). The RNA was then reversed to cDNA by a PrimeScript RT reagent Kit (TaKaRa) (16). SOD2 gene expression was analyzed by PCR and quantitative real-time PCR. The PCR reaction was performed with multiple samples using primers and Premix Taq in accordance to the manufacturer's instruction (TaKaRa) on a Bio-Rad PCR thermocycler (Bio-Rad, California, USA). The reaction products were detected by electrophoresis on a $1.5 \%$ agarose gel containing ethidium bromide. The gel was then photographed under UV transillumination.

QPCR was performed using a master mix containing SuperReal PreMix Plus (TIANGEN, Beijing, China), primers, cDNA and $\mathrm{ddH}_{2} \mathrm{O}$ on a Bio-Rad QPCR thermocycler (Bio-Rad). The changes in SOD2 expression was reported as multiples of increases relative to the aged control group (17). Primers used for SOD2 transcripts detection are as follows: forwards, 5'-GGCTGGCTTGGC TTCAATAA-3'; reverse, 5'-AGCAGTGGAATAAGGCCT GT-3'. Primers used for rat $\beta$-actin transcripts: forward, 5'-CCCATCTATGAGGGTTACGC-3'; reverse, 5'-TTTAATGTCACGCACGATTTC-3'.

Immunohistochemistry (IHC). For immunohistochemistry, the continuous coronal slices of brains were processed in accordance with referential methods (18, 19). Briefly, the slices were dewaxed in graded alcohol. Heated citrate buffer ( $\mathrm{pH}$ 6) was used as antigen retrieval. The slices were blocked by $10 \%$ BSA and then incubated with diluted rabbit anti-SOD2 (diluted $1: 100$, Abcam, Cambridge, UK) overnight at $4^{\circ} \mathrm{C}$. Thereafter the slices were incubated with goat anti-rabbit IgG antibody (HRP-conjugated, diluted $1: 50$, Beyotime, Shanghai, China) for $2 \mathrm{~h}$ at room temperature. Visualization of reaction was observed using Horseradish Peroxidase Color Development Kit (Beyotime) and nuclei were marked using hematoxylin. The sections were sealed with neutral resin and photographed under the microscope.

Statistical analysis. The data were presented as the mean \pm standard (SD). Group differences in the Morris
Water Maze test were analyzed using two-way analysis of variance (ANOVA) with repeated measures with group and training day as the between and within subject factor, respectively. Differences among mean values were analyzed using one-way ANOVA for comparison between treatment groups. $p$-values $\leq 0.05$ were considered statistically significant differences.

\section{RESULTS}

\section{Behavioral alterations}

The behavioral analysis is a sensitive method for revealing the impairment of spatial learning and memory (20). The swimming distance, latency and speed to locate the hidden platform of all rats are shown in Fig. 1 . The test showed that the aged rats took longer to find the hidden platform in second and third days compared with young control group $(p<0.05)$, suggesting that the normal aging rats had significantly cognitive impairment. We found that the prolonged swimming distance and escape latency in the aged control group were shortened by administration of Mde or VitE especially on second day (Fig. 1A, B). Furthermore, the swimming speed of rats in the aged control group was slower than that of the other group (Fig. 1C), but no significant difference was observed either between the aged group and the Mde-treated group or between the aged and young control group $(p>0.05)$. Considered together, these results indicated that Mde prevented aging-induced spatial learning and memory dysfunction in rats, and the effect of Mde was not less than that of the more well-known VitE.

\section{Hematoxylin-eosin staining}

In the young control group, the neurons had normal and clear morphological structure. Cortical cells were arranged in high density (Fig. 2A). The cerebral cortex of the aging rats showed pronounced pathological changes compared to the young rats. A higher number of cells were densely stained, which exhibited pyknotic nuclei and shrinkage of cytoplasm. Additionally, irregularly shaped cells also were seen (Fig. 2B). However, the shrinkage of the neurons was rarely found in the aging rats that treated with high dose of Mde (Fig. 2F). Compared with adult rats, the ratio of neurons in cerebral 

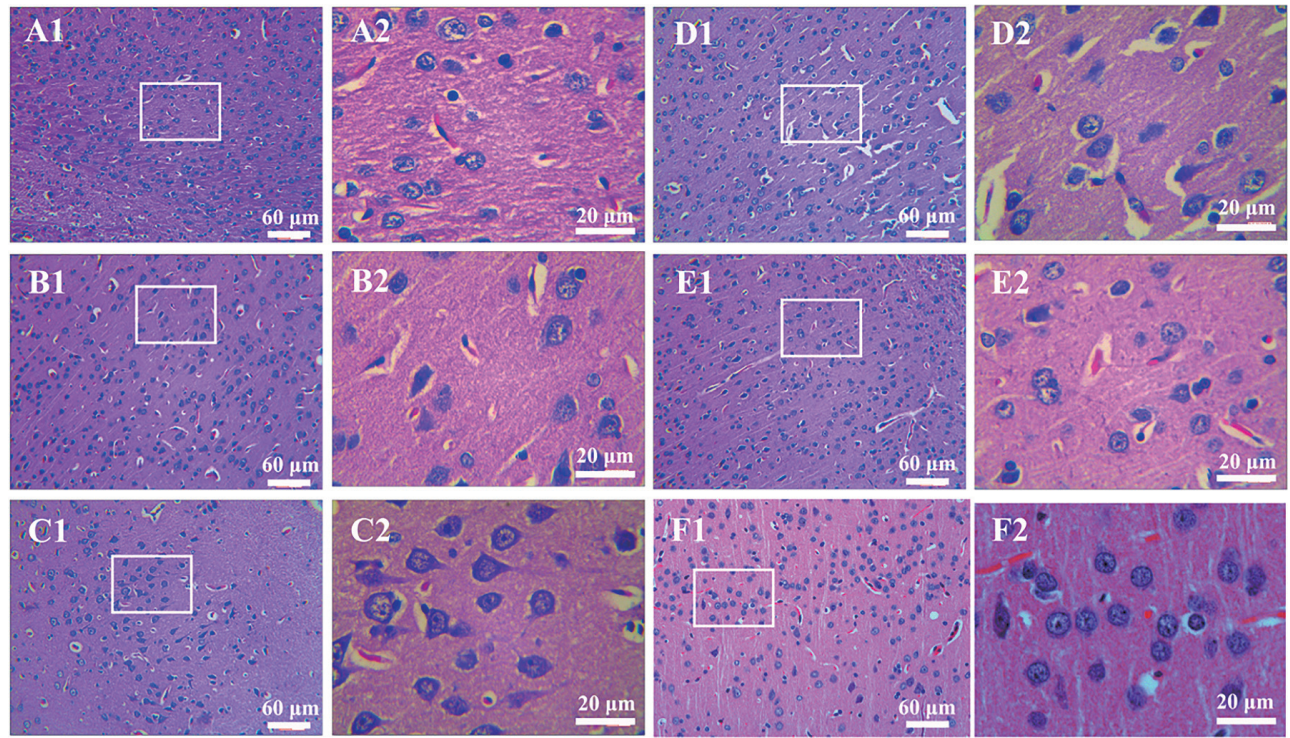

Fig. 2. Representative HE images of cerebral cortex. (A) young control group, (B) aged control group, (C) aged rats treated with VitE, (D) aged rats treated with low-dose Mde, (E) aged rats treated with middle-dose Mde, (F) aged rats treated with high-dose Mde.

Table 1. The quantitative statistics of neuronal parameters in cerebral cortex.

\begin{tabular}{cccccc}
\hline Group & Neuron-density/mm & Cell-density $/ \mathrm{mm}^{2}$ & $\begin{array}{c}\text { Ratio of } \\
\text { neurons/\% }\end{array}$ & $\begin{array}{c}\text { Ratio of damaged } \\
\text { neurons/\% }\end{array}$ & $\begin{array}{c}\text { Max-diameter of } \\
\text { neuronal nuclear/ } \mu \mathrm{m}\end{array}$ \\
\hline A & $107.67 \pm 42.03$ & $4,226.11 \pm 107.67$ & 2.55 & 0 & 13.58 \\
B & $74.02 \pm 25.77$ & $3,425.30 \pm 74.02$ & 2.16 & 0.982 & 10.18 \\
C & $60.57 \pm 20.19$ & $2,839.84 \pm 60.57$ & 2.13 & 0.711 & 11.04 \\
D & $67.29 \pm 11.66$ & $3,297.44 \pm 67.29$ & 2.04 & 0.408 & 10.93 \\
E & $80.75 \pm 38.07$ & $3,930.01 \pm 80.75$ & 2.05 & 0.514 & 10.86 \\
F & $67.29 \pm 34.75$ & $3,082.10 \pm 67.29$ & 2.18 & 0 & 12.75 \\
\hline
\end{tabular}

(A) young control group, (B) aged control group, (C) aged rats treated with VitE, (D) aged rats treated with low-dose Mde, (E) aged rats treated with middle-dose Mde, (F) aged rats treated with high-dose Mde. The main criteria for judging neurons included large cell body, large and round nucleus (diameter $>6.5 \mu \mathrm{m}$ ), shallow staining and obvious nucleolus. The main criteria of damaged neurons included dense staining, pyknotic nuclei and shrinkage of cytoplasm.
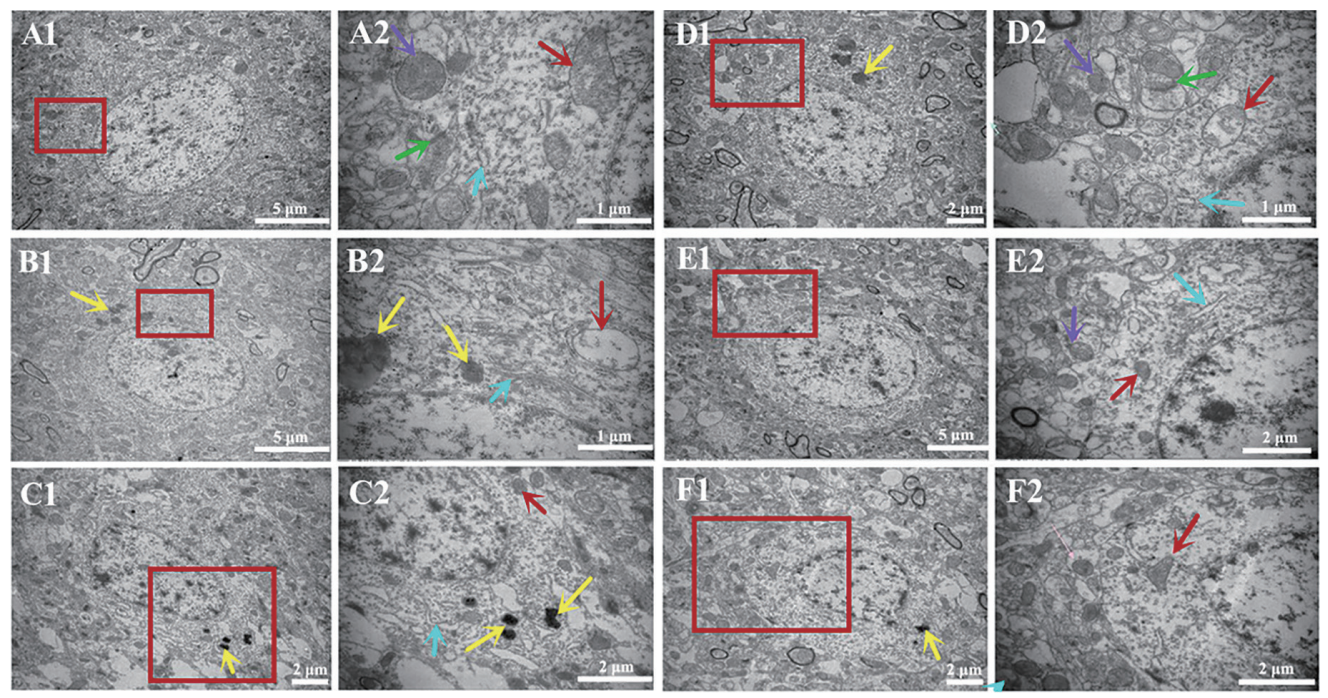

Fig. 3. TEM images of cerebrum neurons after the experiment. (A) young control group, (B) aged control group, (C) aged rats treated with VitE, (D) aged rats treated with low-dose Mde, (E) aged rats treated with middle-dose Mde, (F) aged rats treated with high-dose Mde. Yellow arrows point to the lysosome. Red arrows point to the intracellular mitochondria. Purple arrows point to the extracellular mitochondria. Blue arrows point to the rough endoplasmic reticulum. Green arrows point to the synapses. 
cortex of aging rats has not changed much. Increased proportion of damaged neurons and smaller diameter of neurons was found in aging rats (Table 1). Pathological damage impaired by aging could be prevented from the Mde or VitE and the effect of Mde appeared in a dose-dependent manner (Fig. 2D-E).

Transmission electron microscopy

In the young control group, degenerating neurons were rarely found. The neurons showed intact nuclear with evenly distributed chromatin. The cytoplasm contained intact organelles, such as well-developed mitochondria and rough endocytoplasmic reticulum with rosettes of polyribosomes (Fig. 3A). The synapses of neurons were intact and smooth (Fig. 4A). In the aged control group, neurons had some iconic aging characteristics, consisting of cytoplasmic vacuolization and
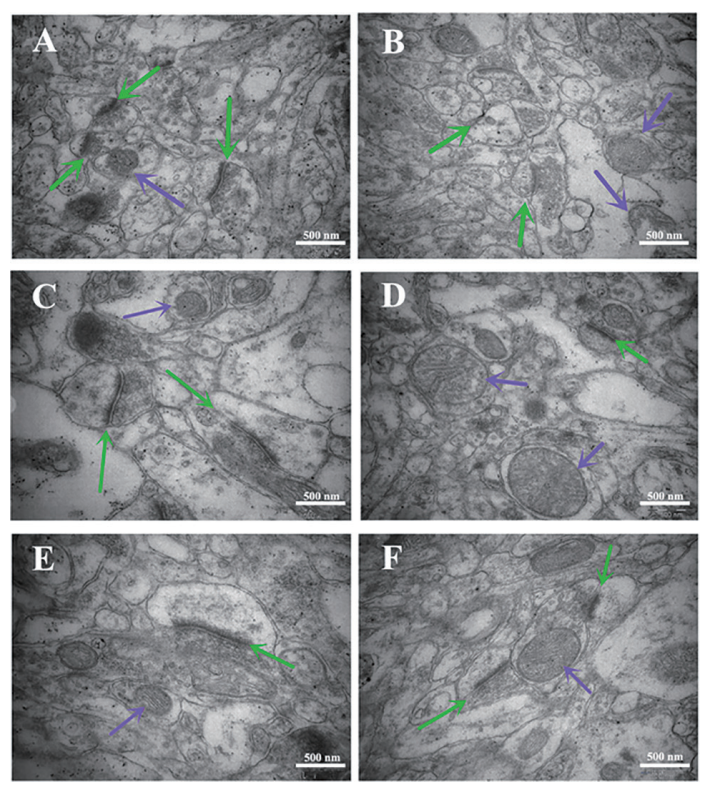

Fig. 4. TEM images of neurons after the experiment. (A) young control group, (B) aged control group, (C) aged rats treated with VitE, (D) aged rats treated with low-dose Mde, (E) aged rats treated with middle-dose Mde, (F) aged rats treated with high-dose Mde. Purple arrows point to the extracellular mitochondria. Green arrows point to the synapses. degenerative changes in the cytoplasmic organelle. Neurons also showed a decrease in their synaptic coverage compared to young controls. Furthermore, endoplasmic reticulum was dilated, fragmented or vacuolated. Ribosomes were obviously detached from the external surface of the rough endoplasmic reticulum. Mitochondrial were swollen without visible cristae. Synaptic gap junction became less and fuzzy. Lots of lysosomes appeared in the cytoplasm of neurons (Figs. 3B, 4B). With the treatment of Mde, the pathological changes had been evidently improved (Fig. 3C-E; Fig. $4 \mathrm{C}-\mathrm{E})$. For example, mitochondrial structure tends to be normal. Lysosomes had fewer numbers in the cytoplasm. The number of neural connections increases. The coloration of synaptosomes was darker. These findings indicated Mde can improve the pathological state of neurons and delay the process of cerebrum senility.

Measurement of biochemical indexes

The oxidative stress proteins (SOD, GPx, MDA) after perfusion with Mde as compared with controls was detected. The SOD and GPx activities in aged control group were found to be lower than young control group (Fig. 5A, C). Compared with the aged control group, the SOD and GPx activities of all Mde-treated rats or VitEtreated rats were found to be significantly increased $(p<0.05)$. Compared with young control group, the MDA content was also easily found to be significantly increased in aged control group (Fig. 5B). Interestingly, Mde or VitE can considerably down-regulate MDA levels in aging rats $(p<0.05)$. In the present study, SOD and GPx activities increased dose-dependently, and the level of MDA decreased in the Mde-treated groups. This phenomenon demonstrates that antioxidant activity is improved and markers of oxidative stress are diminished in Mde-treated aging rats.

PCR, QPCR and IHC

The mRNA content of SOD2 in natural aging rats was significantly decreased compared to young rats (Fig. 6A, B). The expression of SOD2 in aging brain was significantly increased after treatment with Mde $(p<0.001)$. The aging rats with the treatment of Mde reversed the level of SOD2 near to the young rats.

Brown positive particles were predominantly in the
A

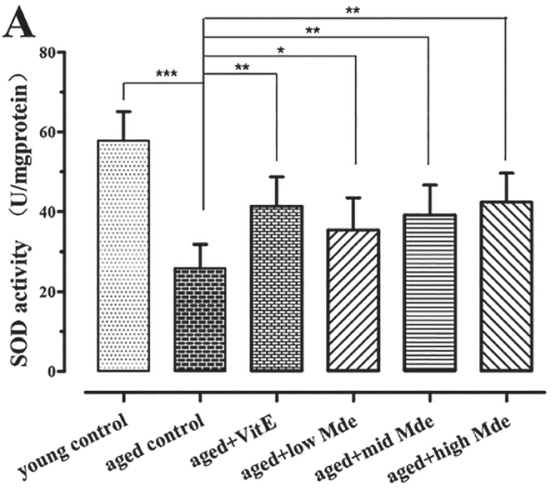

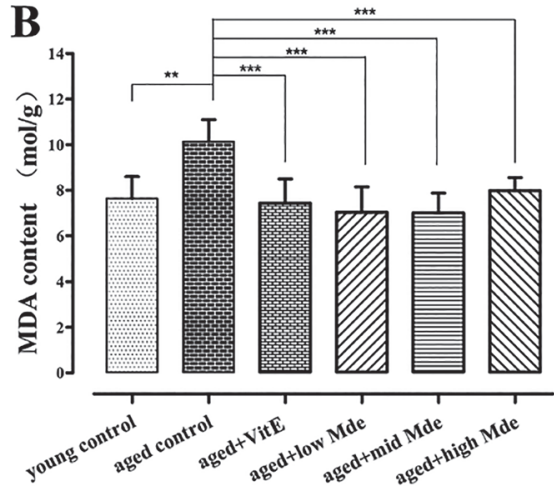

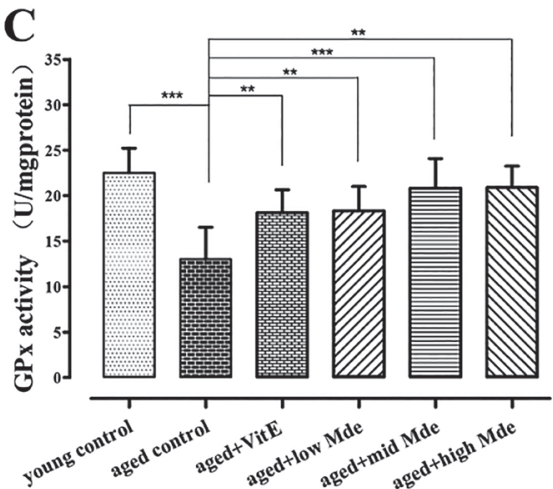

Fig. 5. The SOD, MDA and GPx activity was observed by several biochemical methods. (A) The activity of SOD in the brain. (B) The content of MDA in the brain. (C) The activity of GPx in the brain. Data are represented as mean \pm SD. Other groups were compared with the aged control group, ${ }^{*} p<0.05,{ }^{* *} p<0.01,{ }^{* * *} p<0.001$. 


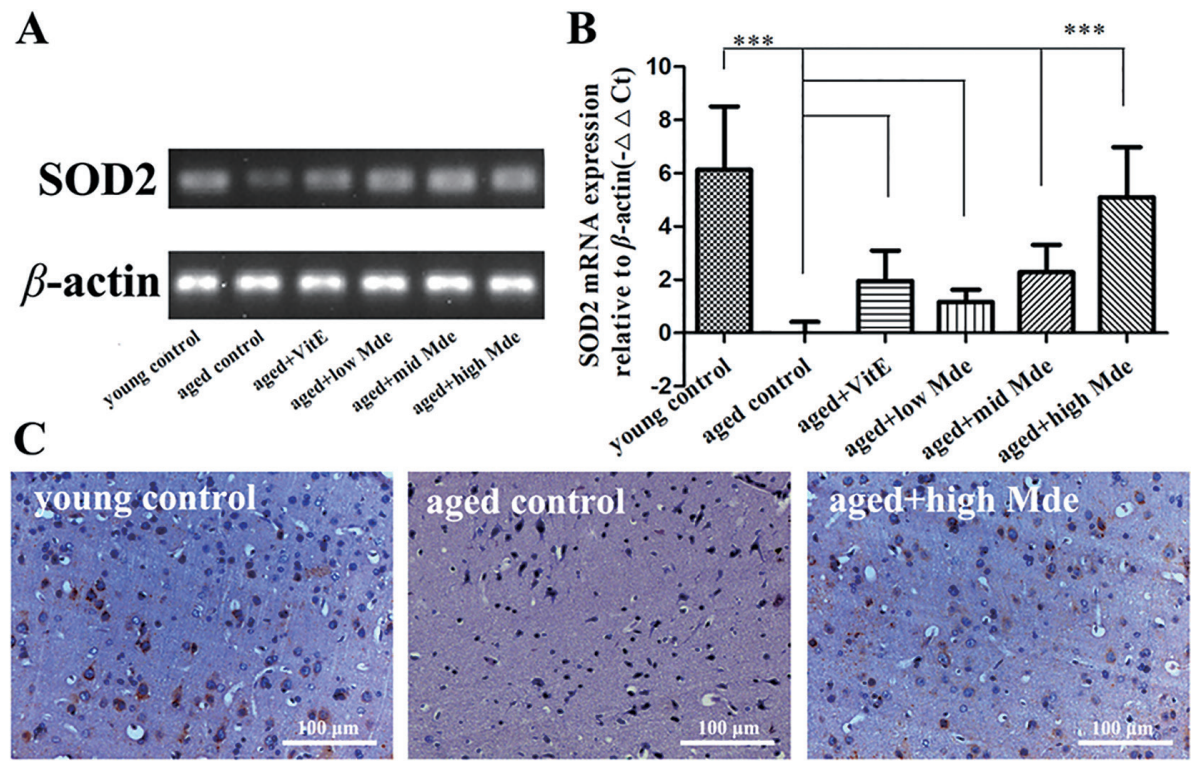

Fig. 6. The SOD2 expression was observed by molecular biology methods. (A) PCR analysis of SOD2 in the brain. (B) QPCR analysis of SOD2 in the brain. QPCR analysis for the ratio SOD2 to $\beta$-actin was performed. Data are represented as mean \pm SD. ${ }^{* * *} p<0.001$. (C) Representative graphs for SOD2 immunohistochemistry.

granular cell layer (GCL) and polymorphic layer (PL). The positive rate of SOD2 was reduced by about $78.27 \%$ in natural aging rats than in young rats. Compared with aged control group, the positive rate of SOD2 was increased by about $65.76 \%$ after perfusion with Mde (Fig. 6C). These phenomena indicated that Mde induce the increase of antioxidant gene and protein.

\section{DISCUSSION}

Natural aging rats are widely used for studying age-related pathogenesis in domestic and foreign laboratory (21). Memory decline is a common phenomenon in the aging process $(22,23)$. The prolongation of escape latency in aged rats in the Morris Water Maze test is thought to reflect age-related learning and memory impairment (24-26). After administration of Mde, the learning and memory of aging rats were significantly improved as evident from the reduced escape latency and swimming distance compared to the aged control group. This is the first report about the improvement of Mde on memory impairment in natural aging rat models.

The cerebral cortex that contains countless nerves and cells controls the minds and actions of the human body. It has been found that aging can reduce the subsets of cerebral neurons and inhibit the proliferation of neurons through immune T cells $(27,28)$. HE staining revealed that the neuronal cells in cerebral cortex were significantly lost and atrophied, and the proportion of damaged neurons increased as compared to adult rats. The changes of neuronal cell density and architecture of neurons were pathological modifications that might happen in the brain in aging processes (29). In addition, the diameter of neurons tends to decrease, and the neuronal cell is shrinking rather than disrupt, which is probably a more decisive age-related alteration (30). It is speculated that Mde can reduce the injury of neurons and is beneficial to the normal growth of neurons. In our study, oral administration of Mde showed a neuroprotective response with significantly attenuated the shrinkage of the neurons and improved organization of cellular layers.

To further investigate the changes of brain ultrastructure, we used the transmission electron microscopy to observe the temporal lobe of neurons. The aging rats exhibited pathological changes including irregularly nuclei in shape and cytoplasmic vacuolization. Furthermore, organelle showed degenerative changes, including lysosomal expansion, mitochondrial edema, endoplasmic reticulum expansion, ribosome shedding and synaptic space stenosis. Decreased cellular function is associated with changes in cell structure. Mitochondrial damage could lead to a decline in hypoxia tolerance, oxidative phosphorylation reaction and protein synthesis. The endoplasmic reticulum and ribosome damage will result in low protein synthesis. The decrease in protein synthesis will affect the cell structure and metabolic processes as well as structural protein and enzyme synthesis. Lysosome can remove aging organelles and useless biological macromolecules. The presence of lysosome is one sign of cellular senescence. Signal transduction between neurons relies on nerve synapses to complete. The abnormalities of information exchange between neurons is closely associated with age-related decline (31). Interestingly, Mde supply was found to attenuate age-associated changes of brain histology and ultrastructure. The structural changes proved Mde has beneficial effect against structural damage in aged brain.

The accumulation of oxygen-centered free radicals (FRs) and other reactive oxygen species (ROS) during aging may contribute to the oxidative damage, which can lead to several events such as loss in protein synthesis, depletion of cellular redox balance and ultimately to 
cell aging and death (32). SOD is the primary substance of free radical scavenging in the organism $(33,34)$. The level of SOD in vivo is an intuitive index about aging and mortality. Glutathione peroxidase $(\mathrm{GPx})$ is an important peroxidative enzyme, which can protect the structure and function of cell membrane from peroxides. Lipid peroxidation end products such as MDA can induce the crosslinking polymerization of macromolecules. Aging rats is characterized by the decreased activity of antioxidant enzymes and increased lipid peroxidation (31). With the treatment of Mde, the antioxidant activity was improved and markers of oxidative stress were diminished in aging rats. These finding indicated that the protection of Mde against age-related neurons damage may be reversed by antioxidants. Moreover, SOD2 display an ability to preserve proliferation of neural stem cells in the aging rats (35). The expression of SOD2 mRNA and protein in brain were examined using PCR, QPCR and immunofluorescence analysis. The results indicated that increased SOD2 in the cerebral cortex may protect the neurons from superoxide-induced damage in Mde-treated aging rats. These results indicated that the protection of Musca domestica larvae extract against age-related memory impairment may be related to attenuation of oxidizing free radical-generated oxidative stress.

It is reported that Musca domestica larvae extract (Mde), the protein-enriched extract of Musca domestica larvae, have significant antioxidant activity (8). In the present study, we found the supplementation of Mde may protect brain from age-related neuronal loss and memory impairment by reducing oxidative stress. The excessive free radical oxidation may damage the normal function of neurons, and ultimately evolve into neurodegenerative diseases $(6,7)$. Brain protection may provide new ideas and application prospects for neurodegenerative diseases (36). In view of the different chemical compositions of VitE and Mde, an appropriate positive control must be necessary in the future studies, such as Tenebrio molitor or Silkworm pupae extract, which contains nutritional value similar to Mde (37, 38 ) and could be better used as a control for delineating specific biological actions of Mde to aged brain. Then, further study need to be carried out to explore the role of Mde in aging-related diseases.

In conclusion, we provide the evidence that the Mde could improve memory impairment and help neurons to maintain normal physiological structure in aging brain. The protective effects of Mde may be realized by reducing oxidative stress and increasing antioxidant enzymes. Musca domestica larvae extract may act as a dietary supplement or developing a medicine to delay brain aging.

\section{Authorship}

Research conception and design: Yanan Tang and Xuemei Lu; experiments: Yanan Tang, Panpan Feng, Shuiqing Gui, and Xuemei Lu; statistical analysis of the data: Xiaobao Jin and Jiayong Zhu; interpretation of the data: Yanan Tang, Panpan Feng and Xuemei Lu; writ- ing of the manuscript: Yanan Tang and Xuemei Lu. All authors discussed the results for completion of the manuscript.

Yanan Tang, Panpan Feng and Shuiqing Gui had equal contribution to this article.

\section{Disclosure of state of COI}

No conflicts of interest to be declared.

\section{Acknowledgments}

This work was supported by the National Natural Science Foundation of China under Grant Number 31501894; Natural Science Foundation of Guangdong Province of China under Grant Number 2018A030313461; and Scientific Research Project of Shenzhen Health and Family Planning System under Grant Number SZXJ2017022.

\section{Supporting information}

Supplemental online material is available on J-STAGE.

\section{REFERENCES}

1) Turnheim K. 2003. When drug therapy gets old: Pharmacokinetics and pharmacodynamics in the elderly. Exp Gerontol 38: 843-853.

2) Dedeyne L, Deschodt M, Verschueren S, Tournoy J, Gielen E. 2017. Effects of multi-domain interventions in (pre)frail elderly on frailty, functional, and cognitive status: A systematic review. Clin Intervent Aging 12: 873896.

3) Shashoua VE, Adams DS, Volodina NV, Li H. 2004. New synthetic peptides can enhance gene expression of key antioxidant defense enzymes in vitro and in vivo. Brain Res 1024: 34-43.

4) Wang JZ, Ding ZQ, Zhang F, Ye WB. 2017. Recent development in cell encapsulations and their therapeutic applications. Mater Sci Eng C Mater Biol Appl 77: 12471260.

5) Wickens PA. 2001. Ageing and the free radical theory. Respir Physiol 128: 379-391.

6) Grimm A, Eckert A. 2017. Brain aging and neurodegeneration: From a mitochondrial point of view. J Neurochem 143: 418-431.

7) Stiegler P, Sereinigg M, Puntschart A, Bradatsch A, Seifert-Held T, Wiederstein-Grasser I, Leber B, Stadelmeyer E, Dandachi N, Zelzer S, Iberer F, Stadlbauer V. 2013. Oxidative stress and apoptosis in a pig model of brain death (bd) and living donation (ld). J Transl Med 11: 244.

8) Zhu L, Wang P, Qin QL, Zhang H, Wu YJ. 2013. Protective effect of polypeptides from larva of housefly (Musca domestica) on hydrogen peroxide-induced oxidative damage in HepG2 cells. Food Chem Toxicol 60: 385-390.

9) Chu FJ, Jin XB, Zhu JY. 2011. Housefly maggots (Musca domestica) protein-enriched fraction/extracts (pe) inhibit lipopolysaccharide-induced atherosclerosis pro-inflammatory responses. J Atheroscler Thromb 18: 282290.

10) Lu X, Jin X, Wang J, Chu F, Zhu J. 2014. Antihepatitis B virus activity of a protein-enriched fraction from housefly (Musca domestica) in a stable HBV-producing cell line. Sci World J 2014: 1-6.

11) Xiao M, Jin X, Tang F, Wang L, Mao J, Li X, Chu F, Huang 
Y, Jing D, Chen W. 2016. Anti-atherosclerotic effect of housefly (Musca domestica) maggot-derived protein-enriched extracts by dampened oxidative stress in apolipoprotein e-deficient mice. RSC Adv 6: 105363-105370.

12) Tian Y, Zou B, Yang L, Xu SF, Yang J, Yao P, Li CM. 2011. High molecular weight persimmon tannin ameliorates cognition deficits and attenuates oxidative damage in senescent mice induced by D-galactose. Food Chem Toxicol 49: 1728-1736.

13) Gao J. 2011. The study of information coding of intrusive memory in posttraumatic stress disorder and its potential pathological mechanism. Third Military Medical University, Chongqing.

14) Golomb E, Matza D, Cummings CA, Schwalb H, Kodavanti UP, Schneider A, Houminer E, Korach A, Nyska A, Shapira OM. 2012. Myocardial mitochondrial injury induced by pulmonary exposure to particulate matter in rats. Toxicol Pathol 40: 779-788.

15) Audrey B, Alexis D, Andrea V, Julio O, Freddy P-P. 2017. Processing and characterization of canine mixed mammary tumor using transmission electron microscopy. Microsc Res Tech 80: 1229-1233.

16) Su J, Jiang L, Wu J, Liu Z, Wu Y. 2016. Anti-tumor and anti-virus activity of polysaccharides extracted from sipunculus nudus (SNP) on hepg2.2.15. Int J Biol Macromolec 87: 597-602.

17) Rong K, Cui Y, Fisher GJ, Xiaojuan Wang BS, Yinbei Chen MS, Schneider LM, Majmudar G. 2015. A comparative study of the effects of retinol and retinoic acid on histological, molecular, and clinical properties of human skin. J Cosmetic Dermatol 15: 49-57.

18) Parabucki AB, Božić ID, Bjelobaba IM, Lavrnja IC, Brkić PD, Jovanović TS, Savić DZ, Stojiljković MB, Peković SM. 2012. Hyperbaric oxygenation alters temporal expression pattern of superoxide dismutase 2 after cortical stab injury in rats. Croatian Med J 53: 586-597.

19) Carvalho MD, De Mesquita JF, Eleutherio EC. 2017. In vivo characterization of i91t sod2 polymorphism of Saccharomyces cerevisiae. J Cell Biochem 118: 10781086.

20) Brandeis R, Brandys Y, Yehuda S. 1989. The use of the morris water maze in the study of memory and learning. Int J Neurosci 48: 29-69.

21) Lee EK, Jang EJ, Jung KJ, Kim DH, Yu BP, Chung HY. 2013. Betaine attenuates lysophosphatidylcholine-mediated adhesion molecules in aged rat aorta: Modulation of the nuclear factor-kappab pathway. Exp Gerontol 48: $517-524$.

22) Biswal S, Sharma D, Kumar K, Nag TC, Barhwal K, Hota SK, Kumar B. 2016. Global hypoxia induced impairment in learning and spatial memory is associated with precocious hippocampal aging. Neurobiol Learning Memory 133: 157-170.

23) Tsai SF, Chen PC, Calkins MJ, Wu SY, Kuo YM. 2016. Exercise counteracts aging-related memory impairment: A potential role for the astrocytic metabolic shuttle. Front Aging Neurosci 8: 1-12.

24) Qu Z, Zhang J, Yang H, Huo L, Gao J, Chen H, Gao W. 2016. Protective effect of tetrahydropalmatine against D-galactose induced memory impairment in rat. Physiol Behav 154: 114-125.

25) Xu J, Shuang R, Xie B, Sun Z, Li Z, Wu H, Ping Y, Zhang X, Zhang Y, Liuab L. 2009. Rejuvenation of antioxidant and cholinergic systems contributes to the effect of procyanidins extracted from the lotus seedpod ameliorating memory impairment in cognitively impaired aged rats. Eur Neuropsychopharmacol 19: 851-860.

26) Hullinger R, Burger C. 2015. Learning impairments identified early in life are predictive of future impairments associated with aging. Behavioural Brain Res 294: 224-233.

27) Dulken BW, Buckley MT, Negredo PN, Saligrama N, Romain Cayrol DSL, Benson M, George SCB, Hebestreit K, Pluvinage JV, Wyss-Coray T, Weissman IL, Vogel H, Davis MM, Brunet A. 2019. Single-cell analysis reveals $\mathrm{T}$ cell infiltration in old neurogenic niches. Nature $\mathbf{5 7 1}$ : 205-225.

28) Soreq L, Rose J, Soreq E, Hardy J, Trabzuni D, Cookson MR, Smith C, Ryten M, Patani R, Ule J. 2017. Major shifts in glial regional identity are a transcriptional hallmark of human brain aging. Cell Reports 18: 557-570.

29) Chen Z, Tang Y, Liu A, Jin X, Zhu J, Lu X. 2017. Oral administration of grifola frondosa polysaccharides improves memory impairment in aged rats via antioxidant action. Molec Nutr Food Res 61: 1-11.

30) Singh R, Kanwar SS, Sood PK, Nehru B. 2011. Beneficial effects of folic acid on enhancement of memory and antioxidant status in aged rat brain. Cell Molec Neurobiol 31: 83-91.

31) Chen B, Zhong Y, Peng W, Sun Y, Kong W-J. 2010. Age-related changes in the central auditory system: Comparison of D-galactose-induced aging rats and naturally aging rats. Brain Res 1344: 43-53.

32) Suliman HB, Piantadosi CA. 2014. Mitochondrial biogenesis: Regulation by endogenous gases during inflammation and organ stress. Curr Pharm Des 20: 56535662.

33) Stiegler P, Sereinigg M, Puntschart A, Bradatsch A, Seifert-Held T, Wiederstein-Grasser I, Leber B, Stadelmeyer E, Dandachi N, Zelzer S, Iberer F, Stadlbauer V. 2013. Oxidative stress and apoptosis in a pig model of brain death (bd) and living donation (ld). J Transl Med 24: 2-12.

34) Yu Y, Li S, Liu Y, Tian G, Yuan Q, Bai F, Wang W, Zhang Z, Ren G, Zhang Y, Li D. 2015. Fibroblast growth factor 21 (FGF21) ameliorates collagen-induced arthritis through modulating oxidative stress and suppressing nuclear factor-kappa b pathway. Int Immunopharmacol 25: 74-82.

35) Ahn JH, Chen BH, Park JH, Kim IH, Cho JH, Lee JC, Yan BC, Choi JH, Hwang IK, Park JH, Han SN, Lee YL, Kim MJ, Won MH. 2014. Hippophae rhamnoides L. leaves extract enhances cell proliferation and neuroblast differentiation through upregulation of intrinsic factors in the dentate gyrus of the aged gerbil. Chin Med J (Engl) 127: 4006-4011.

36) $\mathrm{Wu} \mathrm{Z,} \mathrm{Yu} \mathrm{J,} \mathrm{Zhu} \mathrm{A,} \mathrm{Nakanishi} \mathrm{H.} \mathrm{2016.} \mathrm{Nutrients,}$ microglia aging, and brain aging. Oxidative Med Cellular Longevity 2016: 1-9.

37) Azagoh C, Ducept F, Garcia R, Rakotozafy L, Cuvelier M-E. 2016. Extraction and physicochemical characterization of tenebrio molitor proteins. Food Res Int $\mathbf{8 8}$ : 24-31.

38) Tomotake H, Katagiri M, Yamato M. 2010. Silkworm pupae (Bombyx mori) are new sources of high quality protein and lipid. J Nutr Sci Vitaminol 56: 446-448. 